\title{
Folate pathway gene expression differs in subtypes of acute lymphoblastic leukemia and influences methotrexate pharmacodynamics
}

\author{
Leo Kager,1,2,3 Meyling Cheok, 1,2 Wenjian Yang,,1,2 Gianluigi Zaza,1,2 Qing Cheng,1,2 \\ John C. Panetta, ${ }^{1,2}$ Ching-Hon Pui, ${ }^{1,4,5}$ James R. Downing, ${ }^{1,6}$ \\ Mary V. Relling, ${ }^{1,2,4}$ and William E. Evans ${ }^{1,2,4}$
}

\begin{abstract}
${ }^{1}$ Hematological Malignancies Program, and 2Department of Pharmaceutical Sciences, St. Jude Children's Research Hospital, Memphis, Tennessee, USA. ${ }^{3}$ Department of Hematology-Oncology, St. Anna Children's Hospital, Vienna, Austria. ${ }^{4}$ University of Tennessee, Memphis, Tennessee, USA. ${ }^{5}$ Department of Hematology-Oncology, and ${ }^{6}$ Department of Pathology, St. Jude Children's Research Hospital, Memphis, Tennessee, USA.
\end{abstract}

\begin{abstract}
The ability of leukemia cells to accumulate methotrexate polyglutamate (MTXPG) is an important determinant of the antileukemic effects of methotrexate (MTX). We measured in vivo MTXPG accumulation in leukemia cells from 101 children with acute lymphoblastic leukemia (ALL) and established that B-lineage ALL with either TEL-AML1 or E2A-PBX1 gene fusion, or T-lineage ALL, accumulates significantly lower MTXPG compared with B-lineage ALL without these genetic abnormalities or compared with hyperdiploid (fewer than 50 chromosomes) ALL. To elucidate mechanisms underlying these differences in MTXPG accumulation, we used oligonucleotide microarrays to analyze expression of 32 folate pathway genes in diagnostic leukemia cells from 197 children. This revealed ALL subtype-specific patterns of folate pathway gene expression that were significantly related to MTXPG accumulation. We found significantly lower expression of the reduced folate carrier (SLC19A1, an MTX uptake transporter) in E2A-PBX1 ALL, significantly higher expression of breast cancer resistance protein (ABCG2, an MTX efflux transporter) in TEL-AML1 ALL, and lower expression of FPGS (which catalyzes formation of MTXPG) in T-lineage ALL, consistent with lower MTXPG accumulation in these ALL subtypes. These findings reveal distinct mechanisms of subtype-specific differences in MTXPG accumulation and point to new strategies to overcome these potential causes of treatment failure in childhood ALL.
\end{abstract}

\section{Introduction}

During the past 2 decades, antileukemic agents for the treatment of childhood acute lymphoblastic leukemia (ALL) have remained essentially unchanged; hence the significant increase in event-free survival is attributed largely to optimization of existing medications. Current strategies are aimed at further enhancing efficacy and reducing toxicity of ALL therapy, based on a better understanding of cellular mechanisms of antileukemic effects, and insights into the biological basis of ALL subtype differences in treatment response. Subtypes with a relatively unfavorable prognosis on many treatment protocols include T-lineage ALL (T-ALL) and ALL with rearranged $M L L$ genes or with $B C R-A B L$ gene fusion, whereas ALL with either TEL-AML1 or E2A-PBX1 gene fusions, or hyperdiploid karyotypes (fewer than 50 chromosomes), have a relatively good prognosis with most treatment protocols (1).

\footnotetext{
Nonstandard abbreviations used: $\mathrm{ABCC}, \mathrm{ABC}$, subfamily C; ABCG2, ABC, subfamily G, member 2; ALL, acute lymphoblastic leukemia; ATIC, 5-aminoimidazole4-carboxamide ribonucleotide formyltransferase/IMP cyclohydrolase; BCRP, breast cancer resistance protein; BHD, hyperdiploid B-lineage; BNHD, nonhyperdiploid B-lineage; DHFR, dehydrofolate reductase; DNPS, de novo purine synthesis; FPGS, folylpolyglutamate synthetase; FTH1, ferritin heavy polypeptide 1; GART, phosphoribosylglycinamide formyltransferase; GCH1, GTP cyclohydrolase 1; GGH, $\gamma$-glutamyl hydrolase; HDMTX, high-dose MTX; MRP, multidrug resistance-associated protein; MTHFD, methylenetetrahydrofolate dehydrogenase; MTX, methotrexate; MTXPG, MTX polyglutamate (subscript numbers indicate the number of glutamate residues); PPAT, phosphoribosyl pyrophosphate amidotransferase; RFC, reduced folate carrier; RUVBL2, RuvB, E. coli, homolog-like 2; SLC19A1, solute carrier, family 19, member 1; SVM, support vector machine; T-ALL, T-lineage ALL; TYMS, thymidylate synthetase. Conflict of interest: The authors have declared that no conflict of interest exists.

Methotrexate (MTX) is a major component in all contemporary treatment protocols for childhood ALL, and much is known about the cellular and molecular pharmacology of MTX in ALL (2). The ability of cells to form and accumulate MTX polyglutamates (MTXPGs) is well recognized as a determinant of MTX cytotoxicity $(3,4)$. Compared with MTX, MTXPGs are retained longer in cells (5) and compete more avidly with some cellular folate cofactors (e.g., 10-formyl-tetrahydrofolate, 5,10-methylene-tetrahydrofolate, and 5,10-methenyl-tetrahydrofolate), thereby inhibiting target enzymes in biosynthetic pathways that are critical for DNA synthesis, DNA repair, and cell replication (i.e., thymidine synthesis and de novo purine synthesis [DNPS]) $(6,7)$. In addition to interpatient differences in the systemic pharmacokinetics of MTX (8), cellular MTXPG levels are influenced by (a) transmembrane MTX transport including influx via the reduced folate carrier (RFC, or solute carrier, family 19, member 1 [SLC19A1]), and, to a much lower extent, via folate receptors or low-pH folate transporters $(2,9)$, and active efflux via the $\mathrm{ABC}$ transporters multidrug resistance-associated proteins 1-4 (MRP1-4, or ABCC1-4) (10-12), breast cancer resistance protein (BCRP, or ABCG2) (13), and under certain conditions P-glycoprotein (MDR1, or ABCB1) (14); (b) cytosolic glutamylation of MTX to MTXPG via folylpolyglutamate synthetase (FPGS) (15); and (c) lysosomal degradation of MTXPGs to MTX via $\gamma$-glutamyl hydrolase (GGH) (Supplemental Figure 1; supplemental material available online with this article; doi:10.1172/JCI200522477DS1) (16).

Furthermore, it is likely that other molecules involved in folate and one-carbon metabolism or other cellular proteins can influence MTXPG disposition in leukemia cells. It is well established that 


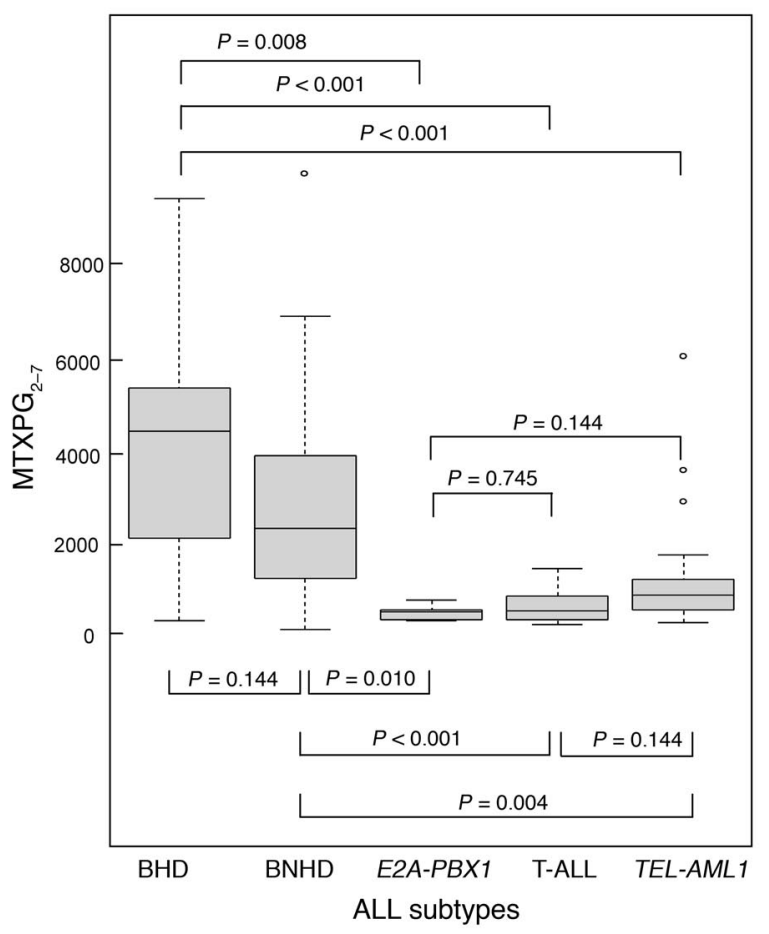

major ALL subtypes accumulate different amounts of MTXPG in vitro (17-19) and in vivo (20), and these differences have been attributed to differences in FPGS activity (21-23), SLC19A1 expression $(24,25)$, and GGH activity (26). However, a comprehensive analysis of gene expression patterns influencing MTXPG accumulation in ALL subtypes has not been previously undertaken. Moreover, there are some ALL subtypes for which intracellular MTXPG disposition has not been thoroughly investigated (e.g., E2A-PBX1 ALL).

The current study was therefore conducted to investigate MTXPG accumulation in specific genetic subtypes of ALL and elucidate mechanisms underlying subtype-specific differences in MTXPG accumulation in leukemia cells after in vivo high-dose MTX (HDMTX) treatment. Such insights hold promise to guide treatment modifications that will further improve outcome of this heterogeneous disease.

\section{Results}

MTXPG accumulation in ALL subtypes and MTX pharmacokinetics. The concentrations of total MTXPG in ALL cells of 101 patients treated with $\operatorname{HDMTX}\left(1 \mathrm{~g} / \mathrm{m}^{2}\right)$, grouped by ALL subtype, are summarized in Figure 1. The groups were B-lineage ALL with TEL-AML1 $(n=24)$, B-lineage ALL with a hyperdiploid karyotype (hyperdiploid B-lineage ALL; BHD ALL; $n=19)$, B-lineage ALL with E2A-PBX1 $(n=5)$, $B$-lineage ALL with no defined molecular genetic abnormality (nonhyperdiploid B-lineage ALL; BNHD ALL; $n=39$ ), and T-ALL $(n=14)$. We found that T-ALL and B-lineage ALL with either the TEL-AML1 or the E2A-PBX1 fusion accumulated significantly lower total and long-chain MTXPG levels after HDMTX, compared with BHD or BNHD ALL $(P<0.0001$; Figure 1). BHD ALL accumulated the highest levels of MTXPG. Similar differences were observed when long-chain MTXPGs were compared (Table 1). There was no difference in MTX plasma concentrations (area under the curve from 0 to 48 hours) among patients with different ALL subtypes ( $P=0.33$, Kruskal-Wallis test).

\section{Figure 1}

Box plot of intracellular concentration of total methotrexate polyglutamates ( $\mathrm{MTXPG}_{2-7}$ ) according to ALL subtypes, following in vivo treatment with $1 \mathrm{~g} / \mathrm{m}^{2}$ MTX infused over 24 hours. MTXPG accumulation (picomoles per $10^{9}$ bone marrow ALL cells) is shown for hyperdiploid B-lineage ALL (BHD, $n=19$ ), nonhyperdiploid B-lineage ALL without defined molecular genetic abnormalities (BNHD, $n=39$ ), ALL with E2A-PBX1 fusion (E2A-PBX1, $n=5)$, T-ALL $(n=14)$, and ALL with TEL-AML1 fusion (TEL-AML1, $n=24$ ). Medians, quartiles, and ranges excluding outliers (circles) are depicted. $P$ values are from pairwise comparisons using the Wilcoxon rank sum test after adjustment for multiple testing.

Gene expression differences across ALL subtypes. After removal of probe sets expressed in less than $5 \%$ of samples and combination of highly correlated probe sets for the same gene (by averaging of standardized signals of each probe set; Pearson correlation coefficient $r>0.4$ ), 25 candidate folate pathway transcripts (corresponding to 23 genes on the HG-U133A GeneChip oligonucleotide microarray; Affymetrix Inc.) were identified (Supplemental Table 1). "Unsupervised" hierarchical clustering of the expression signals of these transcripts clustered patients by ALL subtypes as shown in Figure 2.

T-ALL, TEL-AML1 ALL, BHD ALL, and E2A-PBX1 ALL formed more discrete sample clusters compared with BNHD ALL, demonstrating that these ALL subtypes have distinct patterns of expression of genes involved in folate/antifolate metabolism, whereas there was substantial heterogeneity in BNHD ALL, which has none of these genetic-subtype features. For "supervised" comparisons across subtypes, these 25 transcripts provided an estimated prediction accuracy of $83 \%$ (95\% confidence interval $=75-92 \%)$ for correctly assigning ALL genetic subtype or lineage. The average sensitivity for predicting ALL subtypes based on these 25 transcripts was 93\% for T-ALL, 89\% for TEL-AML1, 84\% for BNHD, 82\% for BHD, and $67 \%$ for $E 2 A-P B X 1$. The average true-positive rate of predicting subtypes was $100 \%$ for T-ALL, $92 \%$ for BHD, $88 \%$ for TEL-AML1, $88 \%$ for $E 2 A-P B X 1$, and $72 \%$ for BNHD. The top 10 folate pathway genes discriminating ALL subtypes are shown in Table 2.

The top 10 discriminating genes include those encoding 2 enzymes involved in DNPS (5-aminoimidazole-4-carboxamide ribonucleotide formyltransferase/IMP cyclohydrolase [ATIC] and phosphoribosylglycinamide formyltransferase [GART]), 3 folate/ antifolate transporters (RFC [SLC19A1], MRP1 [ABCC1], and BCRP [ABCG2]), the enzyme catalyzing folate and MTX polyglu-

\section{Table 1}

In vivo accumulation of total and long-chain MTXPG in ALL cells from 101 children, according to ALL subtypes ${ }^{A}$

\begin{tabular}{lccc}
$\begin{array}{l}\text { ALL } \\
\text { subtype }\end{array}$ & No. & $\begin{array}{c}\text { Total } \text { MTXPG }_{2-7} \\
\text { (pmol/109 cells): } \\
\text { median (range) }\end{array}$ & $\begin{array}{c}\text { Long-chain } \text { MTXPG }_{4-7} \\
\text { (pmol/109 cells): } \\
\text { median (range) }\end{array}$ \\
BHD & 19 & $4,375(377-9,206)$ & $3,170(326-6,525)$ \\
BNHD & 39 & $2,210(186-9,722)$ & $1,702(48-7,736)$ \\
TEL-AML1 & 24 & $911(338-5,906)$ & $602(67-3,456)$ \\
E2A-PBX1 & 5 & $553(364-800)$ & $382(271-628)$ \\
T-ALL & 14 & $572(284-1,468)$ & $355(180-1,096)$ \\
\hline
\end{tabular}

AMeasured 42-44 hours after $1 \mathrm{~g} / \mathrm{m}^{2}$ MTX was infused over 24 hours.

${ }^{B} A L L$ subtypes as defined in Methods and Results. 
tamylation (FPGS), and 2 enzymes catalyzing folate-cofactor interconversion reactions (methylenetetrahydrofolate dehydrogenases 1 and 2 [MTHFD1 and MTHFD2]). The 2 additional genes encode for ferritin heavy polypeptide 1 (FTH1), which may be involved in folate degradation via oxidative catabolism of 5-formyltetrahydrofolate (27), and GTP cyclohydrolase 1 (GCH1), which catalyzes sequential reactions in folate biosynthesis. The expression patterns among ALL subtypes of the 3 MTX transporters and FPGS are depicted in Figure 3; the expression patterns of 4 additional subtype-discriminating folate/antifolate pathway genes (ATIC, GART, MTHFD1, and MTHFD2) are provided in Supplemental Figure 4.

Expression of FPGS was lower in T-ALL compared with all other subtypes (versus BHD, BNHD, and $E 2 A-P B X 1, P<0.0001$; versus TEL-AML1, $P=0.0004$ ) and lower in TEL-AML1 compared with all other subtypes except T-ALL (versus BNHD, $P<0.0001$; versus $B H D, P=0.054$; versus $E 2 A-P B X 1, P=0.060)$. Reduced folate carrier mRNA (RFC, or SLC19A1) was expressed at a higher level in BHD ALL (versus any other ALL subtype, $P<0.0001$ ) and at a lower level in E2A-PBX1 (versus BHD and T-ALL, $P<0.0001$; versus $B N H D, P=0.0006$; versus $T E L-A M L 1, P=0.044)$. In addition, multidrug resistance-associated protein $1 \mathrm{mRNA}(M R P 1$, or $A B C C 1$ ) was expressed at a lower level in E2A-PBX1 ALL (versus BNHD, TEL-AML1, and T-ALL, $P<0.0001$; versus BHD, $P=0.0004$ ), but at a higher level in T-ALL (versus any other ALL subtype, $P<0.0001$ ). The ABCG2 gene, which encodes for an efflux transporter (i.e., BCRP), was found to be overexpressed in TEL-AML1 ALL (versus any other ALL subtype, $P<0.0001$ ). All $P$ values are from pairwise comparisons using the Wilcoxon rank sum test adjusted for multiple testing by Holm's method.

Correlation structure in expression of genes in MTXrelated pathways. Unsupervised hierarchical clustering analysis grouped the 25 transcripts into 2 major clusters, each of which contained functionally interrelated genes (Figure 2). The smaller cluster, highlighted in purple ( 9 transcripts; Figure 2, left), contained most of the transporters (all except $R F C$, or $S L C 19 A 1$, and $M R P 1$, or $A B C C 1$ ), plus genes encoding enzymes catalyzing polyglutamate synthesis (FPGS) or degradation $(G G H)$, whereas the larger cluster (16 transcripts) contained primarily genes involved in folate-mediated one-carbon metabolism and folate

\section{Figure 2}

Unsupervised hierarchical clustering of 25 candidate gene transcripts based on expression in bone marrow ALL cells from 197 newly diagnosed patients with defined ALL subtypes. Each column represents a gene probe set and each row a patient. ALL subtypes are depicted by different colors: red (BHD, $n=42$ ), blue (BNHD, $n=58$ ), yellow ( $E 2 A-P B X 1, n=21)$, black (T-ALL, $n=35)$, and green (TEL-AML1, $n=41)$. The gene cluster highlighted in magenta indicates 7 tightly clustered transcripts. The gene cluster highlighted in purple includes most of the transporters ( 9 transcripts). Probe set signal values were normalized to the mean across patients, and values for each individual case are represented by a color, with green corresponding to SD $(\sigma)$ below and red corresponding to SD $(\sigma)$ above the mean, according to the scale shown. synthesis. Transcripts encoding for enzymes contiguous in folate coenzyme interconversion reactions (MTHFD1, MTHFD2), DNPS (GART, ATIC), and pyrimidine synthesis (thymidylate synthetase [TYMS], dehydrofolate reductase [DHFR]) constituted a tight subcluster (Figure 2, right, highlighted in magenta). Within this subcluster, 1 additional gene was found (RUVBL2), encoding the human homolog of the bacterial (E. coli) DNA helicase RuvB. The expression levels of DHFR, TYMS, MTHFD1, MTHFD2, ATIC, GART, and RUVBL2 strongly correlated with each other (median Pearson correlation coefficient $r=0.44$, range $0.18-0.68$; Figure 4).

Even after adjustment of expression levels for subtype differences, these 7 transcripts still constituted a tight cluster (data not shown). A TRANSFAC database search for transcription factor binding sites within 1,200 bp upstream of exon 1 was performed for all 25 transcripts, revealing E2F binding sites in all 7 transcripts whose expression levels were well correlated (i.e., DHFR, -11 bp; TYMS, -8 bp; GART, -262 bp; MTHFD1, -285 bp; ATIC, -368 bp; MTHFD2, -913 bp; and RUVBL2, $-1,142$ bp), but not in the other transcripts.

Association between gene expression and cellular MTXPG accumulation. Because total MTXPG accumulation was significantly correlated with ALL subtypes $\left(r^{2}=0.56, P<0.0001\right.$, linear regression model), we investigated whether the expression of these 25 transcripts was sig-

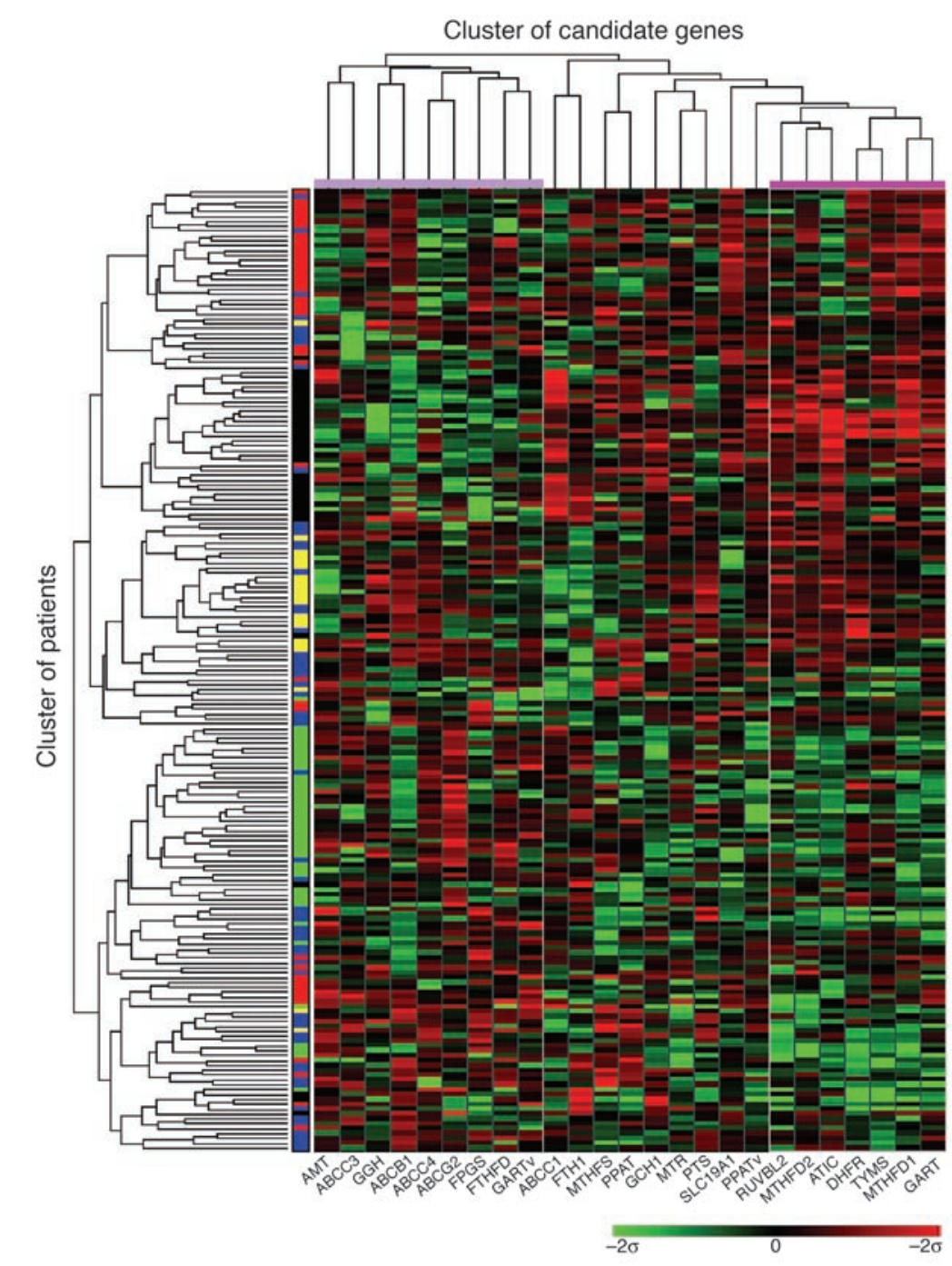


Table 2

Top discriminating candidate genes whose expression was significantly related to ALL subtype ${ }^{A}$

\begin{tabular}{|c|c|c|c|c|c|c|c|c|}
\hline \multirow[b]{2}{*}{ Gene symbol } & \multirow[b]{2}{*}{ Probe set ID } & \multicolumn{5}{|c|}{ Median gene expression signal } & \multirow[b]{2}{*}{ Gene product } & \multirow[b]{2}{*}{ Function } \\
\hline & & BHD & BNHD & $E 2 A-P B X 1$ & T-ALL & TEL-AML1 & & \\
\hline ATIC & 208758_at & 5,318 & 8,908 & 9,701 & 12,702 & 5,551 & $\begin{array}{c}\text { 5-Aminoimidazole-4-carboxamide } \\
\text { ribonucleotide formyltransferase/ } \\
\text { IMP cyclohydrolase }\end{array}$ & DNPS \\
\hline$A B C C 1$ & $\begin{array}{l}\text { 202804_atB } \\
202805 \_s \_a t^{B}\end{array}$ & 3,502 & 4,780 & 1,693 & 18,038 & 6,612 & ABC, subfamily $\mathrm{C}$, member 1 (MRP1) & Transporter \\
\hline SLC19A1 & 211576_s_at & 3,413 & 1,721 & 1,077 & 2,104 & 1,462 & Solute carrier, family 19, member 1 (RFC) & Transporter \\
\hline MTHFD1 & 202309_at & 7,385 & 5,505 & 5,748 & 8,518 & 4,185 & Methylenetetrahydrofolate dehydrogenase 1 & $\begin{array}{l}\text { One-carbon } \\
\text { metabolism }\end{array}$ \\
\hline ABCG2 & 209735_at & 1,195 & 1,597 & 1,378 & 960 & 3,079 & ABC, subfamily G, member 2 (BCRP) & Transporter \\
\hline FPGS & 202945_at & 6,207 & 7,490 & 6,346 & 3,647 & 5,358 & Folylpolyglutamate synthetase & $\begin{array}{l}\text { Polyglutamylation } \\
\text { of folates/antifolates }\end{array}$ \\
\hline FTH1 & 200748_s_at ${ }^{B}$ & 34,919 & 16,390 & 12,068 & 41,353 & 34,020 & Ferritin heavy polypeptide 1 & $\begin{array}{l}\text { One-carbon } \\
\text { metabolism }\end{array}$ \\
\hline MTHFD2 & $\begin{array}{l}\text { 214211_at' } \\
\text { 201761_at }\end{array}$ & 3,922 & 3,680 & 4,156 & 5,473 & 2,037 & Methylenetetrahydrofolate dehydrogenase 2 & $\begin{array}{l}\text { One-carbon } \\
\text { metabolism }\end{array}$ \\
\hline GCH1 & 204224_s_at & 2,648 & 2,660 & 2,873 & 3,098 & 1,281 & GTP cyclohydrolase 1 & Folate synthesis \\
\hline GART & $\begin{array}{l}\text { 212378_atB } \\
212379 \_a t^{B}\end{array}$ & 4,577 & 2,312 & 2,000 & 3,309 & 1,136 & Phosphoribosylglycinamide formyltransferase & DNPS \\
\hline
\end{tabular}

AP $<0.0001$, Kruskal-Wallis test. ${ }^{B}$ Highly correlated probe sets for the same gene were combined into 1 expression variable.

nificantly predictive of total MTXPG accumulation in a leave-oneout cross-validation with support vector machine (SVM). We constructed SVMs with both linear and nonlinear (radial) kernels, the latter reflecting more complex interactions among these transcripts. The predicted total MTXPG levels were significantly correlated with the observed MTXPG $\left(r^{2}=0.21, P=0.0004\right.$, linear kernel; $r^{2}=0.37$, $P<0.0001$, nonlinear kernel). In multiple linear regression analyses that included ALL subtype as a covariate, the MTXPG values predicted based on the nonlinear kernel remained significantly related to the observed MTXPG levels ( $P=0.043$ ), whereas the MTXPG values based on the linear kernel were no longer significant $(P=0.79)$.

Among patients with both MTXPG accumulation and gene expression data $(n=54$; BNHD, $n=22$; BHD, $n=9$; T-ALL, $n=9 ; T E L-A M L 1, n=11 ; E 2 A-P B X 1, n=3)$, the largest subgroup was BNHD, which also exhibited the highest variation in total MTXPG accumulation. Spearman's rank correlation between MTXPG and gene expression within this group of patients revealed that the expression of TYMS $(\rho=0.65, P=0.0014)$, $\operatorname{MTHFD} 1(\rho=0.62, P=0.0027)$, and RUVBL2 $(\rho=0.60, P=0.0036)$ significantly discriminated total MTXPG accumulation in BNHD ALL. The expression of TYMS remained statistically significant even after adjustment for multiple testing (adjusted $P=0.025)$. The correlation between total MTXPG accumulation and expression of TYMS and MTHFD1 is depicted in Supplemental Figures 5 and 6. MTHFD2 $(\rho=0.74, P=0.025), P P A T(\rho=-0.73$, $P=0.03)$, and RUVBL2 $(\rho=-0.69, P=0.05)$ were predictive of MTXPG accumulation within T-ALL. None of the transcripts was predictive of variability in MTXPG accumulation within BHD or TEL-AML1 ALL, likely because of the small number of patients in these 2 subgroups.

DNA synthesis and folate pathway gene expression. Data on the percentage of cells in $S$ phase of the cell cycle and gene expression were available in 52 patients (BHD, $n=8$; BNHD, $n=18$; E2A-PBX1, $n=6$; T-ALL, $n=4$; TEL-AML1, $n=16$ ). There were no significant differences in the percentage of cells in S phase among ALL subtypes ( $P=0.17$, Kruskal-Wallis test). By Spearman's rank correlation, mRNA levels of TYMS $(\rho=0.57, P<0.0001)$, DHFR $(\rho=0.51$, $P=0.0001)$, MTHFD1 $(\rho=0.30, P=0.029)$, and RUVBL2 $(\rho=0.28$, $P=0.04)$ were significantly associated with the percentage of cells in $S$ phase (Supplemental Figure 7).

Folate pathway gene expression, MTXPG accumulation, and peripheral blast reduction after HDMTX. Cellular MTXPG accumulation in BNHD ALL cells ( $n=14$ patients) was significantly correlated with MTX's in vivo cytotoxic effect, as measured by the reduction of circulating ALL cells over 4 days following HDMTX therapy $(\rho=0.6$, $P=0.026, n=14$, Spearman's rank correlation). Within this group of ALL patients, ABCG2 mRNA expression was significantly correlated with MTX's in vivo cytotoxic effect $(\rho=-0.68, P=0.009)$; in addition, mRNA levels of ABCC4 $(\rho=-0.52, P=0.06)$, TYMS $(\rho=0.49, P=0.07)$, and ATIC $(\rho=-0.48, P=0.087)$ were marginally correlated with peripheral blood blast reduction on day 4 .

\section{Discussion}

MTXPG accumulation in ALL cells is a determinant of MTX's in vivo antileukemic effects (3). MTXPG accumulation has been previously shown to be lower in T-ALL and higher in BHD ALL, compared with BNHD ALL, both in vivo $(3)$ and ex vivo $(17,18)$. In addition, ALL cells carrying the TEL-AML1 fusion gene have been shown to accumulate lower MTXPG levels in vitro, when compared with B-lineage cells without this translocation (19), and the current work has documented this in vivo. In addition to validating lower accumulation of MTXPG in T-ALL and TEL-AML1 ALL in vivo, we identified ALL with the E2A-PBX1 fusion as a new ALL subtype with significantly lower MTXPG accumulation. Furthermore, our candidate-gene strategy to identify folate pathway genes related to MTXPG accumulation has revealed new insights into mechanisms underlying these lineage and genetic-subtype differences in MTXPG accumulation in ALL cells. Our results indicate 
A
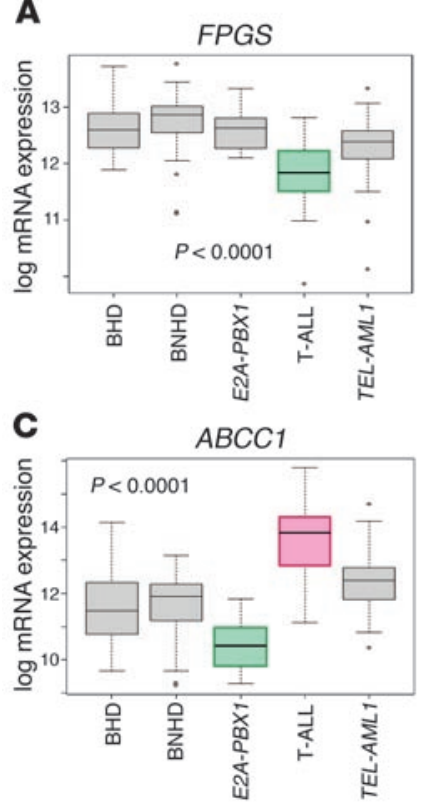

B

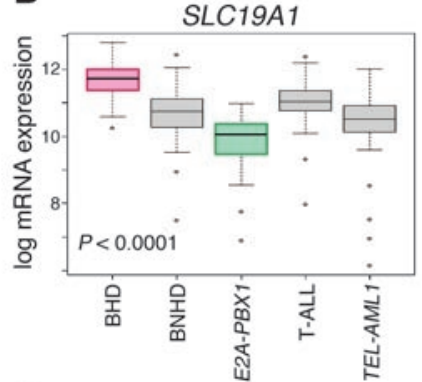

D

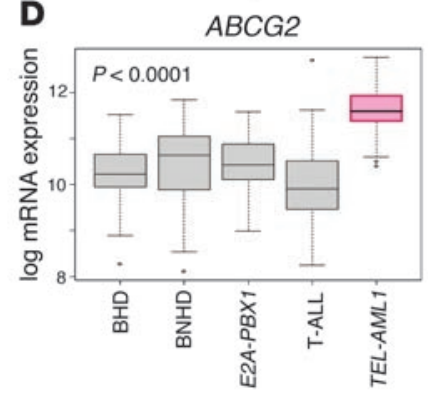

that genes encoding for proteins involved in cellular folate/antifolate homeostasis have distinct expression patterns across the 5 ALL subtypes studied (Figure 2), and that the pattern of expression of these genes is significantly related to MTXPG accumulation in ALL cells. Interestingly, the correlation between MTXPG accumulation and gene expression pattern was better described using a nonlinear model, which likely reflects the complexity of interaction among the selected candidate genes, and the nonlinear nature of many biological processes.

Among the folate pathway genes whose expression was significantly associated with ALL subtype, 2 genes have well-defined functions in cellular MTX uptake and polyglutamylation: reduced folate carrier (RFC, or SLC19A1) and FPGS. FPGS expression was significantly lower in T-ALL, compared with other subtypes, a finding that is in line with our previous report of lower FPGS activity in T-ALL $(21,22)$, which has subsequently been corroborated by others (23). We have also previously reported that lymphoblast FPGS activity is significantly correlated with FPGS mRNA levels (22); therefore, the lower FPGS expression observed in ALL cells containing the TEL-AML1 fusion gene (Figure $3 \mathrm{~A}$ ) points to this as a mechanism for low MTXPG accumulation in this ALL subtype as well.

We and others have shown that the SLC19A1 gene, which encodes the predominant MTX (and folate) uptake transporter, $\mathrm{RFC}$, is overexpressed in B-lineage cells with more than 50 chromosomes $(24,25)$, partly because of extra copies of chromosome 21 , and that its overexpression is associated with high cellular accumulation of MTXPG (25). Our current findings have identified an opposite phenotype in ALL cells containing the E2A-PBX1 gene fusion, which exhibit significantly lower SLC19A1 expression and MTXPG accumulation. This gene fusion, which results from a $\mathrm{t}(1 ; 19)$ ( $\mathrm{q} 23 ; \mathrm{p} 13.3)$ chromosomal translocation, is found in about $5 \%$ of children and adults with $\operatorname{ALL}(1,28)$. Children whose leukemic cells express $E 2 A-P B X 1$ have a poor prognosis compared with patients with standard-risk B-lineage ALL, when treated with conventional antimetabolite-based chemotherapy protocols $(29,30)$.

\section{Figure 3}

Gene expression of FPGS and 3 transporters with known MTX transport capacity in ALL subtypes. Box plots with medians, quartiles, and ranges excluding outliers (circles) of log mRNA expression are depicted for folylpolyglutamate synthetase (FPGS) (A), reduced folate carrier (RFC, or SLC19A1) (B), multidrug resistance-associated protein 1 $(M R P 1$, or $A B C C 1)(\mathbf{C})$, and breast cancer resistance protein (BCRP, or $A B C G 2$ ) (D). Data from 197 patients were plotted (BHD, $n=42$; BNHD, $n=58$; E2A-PBX1, $n=21$; T-ALL, $n=35$; TEL-AML1, $n=41$ ). $P$ values were determined by the Kruskal-Wallis test. The red boxes indicate subtypes in which gene expression was significantly higher, whereas the green boxes indicate subtypes with significantly lower expression of the gene depicted.

Empirically based treatment intensification, including consolidation therapy with HDMTX, has resulted in improved cure rates in children with $E 2 A-P B X 1$ ALL $(1,31)$, but it is unknown which intensification element(s) is responsible for the improved outcome. The current finding of low MTXPG accumulation in E2A$P B X 1$ ALL is the first chemotherapy-resistance mechanism to be identified in this ALL subtype. As impaired drug transport via SLC19A1 is a documented mechanism of MTX resistance in ALL $(32,33)$ and $S L C 19 A 1$ expression in E2A-PBX1 ALL is low, this suggests that HDMTX may be a strategy to overcome resistance and therefore improve outcome in this ALL subtype (34). Indeed, we observed that in vivo MTXPG accumulation in E2A-PBX1-positive bone marrow cells obtained 42-44 hours after HDMTX was significantly higher in 5 children who received HDMTX $\left(1 \mathrm{~g} / \mathrm{m}^{2}\right)$ infused over 24 hours compared with 6 children who received low-dose MTX (i.e., $30 \mathrm{mg} / \mathrm{m}^{2}$ orally every 6 hours, 6 times; median 553 versus 246 pmol $\mathrm{MTXPG}_{2-7}$ per $10^{9}$ cells, $P=0.017$, Wilcoxon rank sum test). Additional studies are needed to define the nature of SLC19A1 function and MTX uptake as a resistance mechanism in E2A-PBX1 ALL. However, our results indicate that HDMTX consolidation therapy may be of particular benefit for this subgroup of children with ALL. Notably, the clinical trials
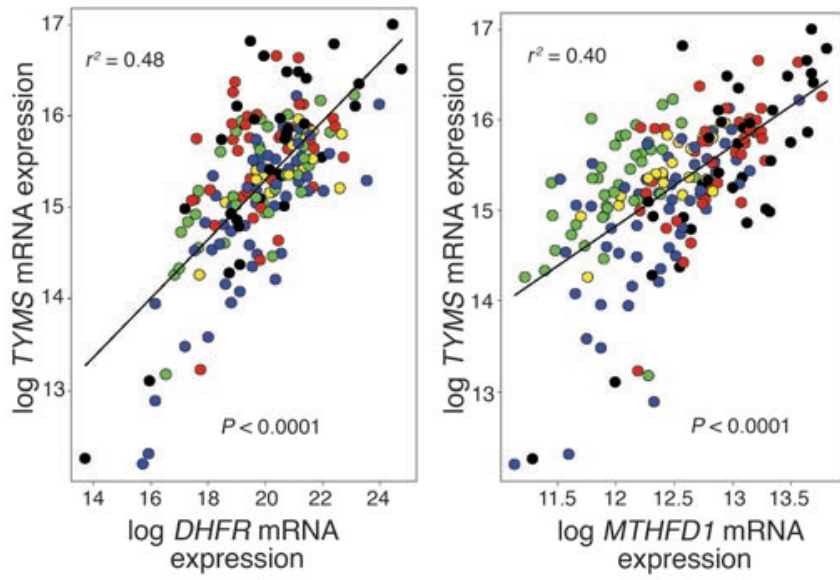

\section{Figure 4}

Correlation in mRNA expression levels in ALL cells between DHFR and TYMS, and between TYMS and methylenetetrahydrofolate dehydrogenase 1 (MTHFD1). Data are shown for ALL cells from 197 patients. $P$ values are from Pearson correlation. BHD subtypes $(n=42)$ are depicted in red, BNHD $(n=58)$ in blue, E2A-PBX1 $(n=21)$ in yellow, T-ALL $(n=35)$ in black, and TEL-AML1 $(n=41)$ in green. 
with the best treatment results for E2A-PBX1 ALL, the St. Jude Total Therapy XIII protocol and the Berlin-Frankfurt-Muenster (ALL-BFM 90) protocol, with 5-year event-free survival rates of $89.5 \% \pm 7.3 \%$ and $93 \% \pm 6 \%$, respectively, featured HDMTX consolidation therapy $(1,35)$.

Two other genes whose expression was significantly different among ALL subtypes also encode MTX transporters, $A B C C 1$ and $A B C G 2$. The $A B C C 1$ product is multidrug resistance-associated protein 1 (MRP1), an $A B C$, subfamily $C$, cellular efflux pump. In shortterm exposure assays, $A B C C 1$ overexpression can confer resistance to MTX by mediating cellular MTX efflux, resulting in lower cellular accumulation and less substrate for polyglutamylation $(10,11)$. Although ABCC1 lowered cellular MTX and MTXPG accumulation in long-term exposure assays as well, MTX resistance was not observed under this condition, and the limited-resistance phenotype has been attributed to the inability of MRPs to transport MTXPGs (10). However, the impact of $A B C C 1$ on cellular MTXPG accumulation and MTX resistance is complex, as $A B C C 1$ plays an important role in cellular folate homeostasis as well (36-38). We observed overexpression of $A B C C 1 \mathrm{mRNA}$ in T-ALL, but very low expression in E2A-PBX1 ALL, yet both ALL subtypes accumulate low MTXPG after $1 \mathrm{~g} / \mathrm{m}^{2}$ MTX infused over 24 hours (i.e., longterm MTX exposure). This may be explained by differences in compensatory mechanisms in these ALL subtypes, such as lower RFC (SLC19A1) expression in E2A-PBX1 but not T-lineage ALL. Further studies will be required to better define the importance of $A B C C 1$ in MTXPG and folate accumulation in leukemic cells.

Interestingly, $A B C G 2$, which encodes for $A B C$, subfamily $G$, member 2 (i.e., BCRP), was overexpressed in ALL cells containing the TEL-AML1 gene fusion (Figure 3D). ABCG2 is a low-affinity, high-capacity MTX transporter, involved in MTX efflux from cells (13). In contrast to MRPs, ABCG2 additionally transports short-chain MTXPGs, which has been proposed as the underlying mechanism for MTX resistance in ABCG2-overexpressing cell lines after continuous MTX exposure (13). It is noteworthy that only wild-type BCRP, but not the mutant forms (R482T or R482G), transports MTX and MTXPGs $(13,39)$. A recent study on primary ALL cells from 46 newly diagnosed patients (children and adults) showed that $A B C G 2$ was more highly expressed and functionally more active in B-lineage ALL than in T-ALL (40), which is in line with our findings (Figure 3D). However, our findings extend these early findings by showing that a specific genetic subtype of B-lineage ALL (TEL-AML1) has significantly higher expression of ABCG2 than all other ALL subtypes.

Though it was not the primary objective of our study, we established distinct correlation structures in gene expression patterns among the candidate folate pathway genes in ALL cells. Of note, 7 genes, i.e., DHFR, TYMS, MTHFD1, MTHFD2, ATIC, GART, and RUVBL2, constituted a tight subcluster. DHFR and TYMS expression is controlled by E2F-family transcription factors; and there is evidence that cell cycle entry and proliferation are regulated by the balance of E2F activities (41). Correlated expression of DHFR and TYMS has been observed in sarcoma cell lines (42) and has been documented in ALL cells via quantitative RT-PCR as well (43). We detected E2F binding sites within the promoter region of all 7 genes in this cluster of functionally related genes, but not in the other 18 folate pathway genes investigated.

Our results indicate that in ALL cells, the expression of genes encoding enzymes contiguous in one-carbon interconversion reactions (MTHFD1 and MTHFD2), DNPS (GART and ATIC), and pyrimidine synthesis (TYMS and DHFR) is highly correlated, consistent with common regulation of their expression. Interestingly, GART, ATIC, MTHFD1, MTHFD2, and RUVBL2 are expressed at a low level in TEL-AML1 ALL, which is in line with our recent finding of lower DNPS in this ALL subtype (44). As expected, MTHFD1 expression was highly correlated with cellular DNPS in 48 diagnostic ALL samples ( $r^{2}=0.34, P<0.0001$; data not shown). Conversely, these 7 genes were overexpressed in T-ALL. Patients with T-ALL often present with high blast counts at diagnosis (45); therefore the overexpression of key enzymes of pyrimidine and de novo purine synthesis is in line with significantly higher DNPS rates in T-ALL (46). In addition, we established that TYMS, DHFR, MTHFD1, and RUVBL2 expression is significantly correlated with the percentage of ALL cells in S phase (Supplemental Figure 7).

The current study confirmed previous findings (3) showing that MTXPG accumulation is a determinant of MTX's in vivo antileukemic effect, as measured by the reduction of circulating ALL cells on day 4 after HDMTX therapy, in the largest ALL subgroup (BNHD; $\rho=0.6, P=0.026, n=14$, Spearman's rank correlation). Furthermore, we found a significant relationship between expression of ABCG2, ABCC4, and TYMS and MTX's cytotoxic effects (i.e., reduction in circulating blasts on day 4 after HDMTX) in BNHD ALL.

The current study documented that MTXPG accumulation differs significantly among the 5 ALL subtypes studied and identified E2A-PBX1 ALL as a new subtype with low MTXPG accumulation. To our knowledge, our studies also provide the first evidence of ALL subtype-specific expression patterns for folate pathway genes. Moreover, the pattern of expression of genes known to be involved in MTX uptake or efflux from ALL cells (e.g., SLC19A1, ABCC1, $A B C G 2)$ and MTX polyglutamylation (FPGS) discriminated the observed ALL subtype differences in MTXPG accumulation. The current work has thus revealed new insights into the mechanisms responsible for ALL subtype differences in MTXPG accumulation, identified ALL with the E2A-PBX1 fusion as a new genetic subtype with low MTXPG accumulation, and pointed to ALL subtype-specific strategies to overcome this cause of ALL drug resistance.

\section{Methods}

Patients, ALL subtypes, and treatment. A total of 244 children aged 18 years or younger with newly diagnosed ALL, enrolled in the Total Therapy XIIIA, $\mathrm{XIIIB}$, and XV protocols, were included in this study (31) (Supplemental Figure 2). The Institutional Review Board at St. Jude Children's Research Hospital approved the investigation, and signed informed consent was obtained from patients, parents, or legal guardians before enrollment, as appropriate. The diagnosis of ALL was based on morphology, cytochemical staining, immunophenotyping, cytogenetic analysis, and molecular characterization, as previously described $(1,47)$. All patients had well-characterized ALL subtypes and were grouped into B-lineage leukemia that contained $t(1 ; 19)$ $(E 2 A-P B X 1, n=23), \mathrm{t}(12 ; 21)(T E L-A M L 1, n=46)$, a hyperdiploid karyotype (i.e., > 50 chromosomes; $\mathrm{BHD}, n=52$ ), or neither any of these nor any other prognostic molecular genetic abnormalities (BNHD, $n=75$ ); and T-ALL $(n=48)$. Because of the small number of patients with $M L L$-rearranged or $B C R-A B L$ ALL, these subtypes were not included in our analysis.

The amount of MTXPG in bone marrow ALL cells was measured at 42-44 hours after treatment in 101 patients, who received identical singleagent HDMTX treatment, according to either the Total Therapy XIIIA $(n=56)$ or the Total Therapy XV $(n=45)$ protocol. MTX was given as a $200 \mathrm{mg} / \mathrm{m}^{2}$ i.v. push, followed by $800 \mathrm{mg} / \mathrm{m}^{2}$ continuous i.v. infusion over 24 hours, after prehydration and under supportive therapy as previously described (3). Leucovorin rescue was started after the 42- to 44-hour bone 
marrow aspirates were obtained. Samples consisted of 5-10 $\mathrm{ml}$ of bone marrow collected in syringes containing $800 \mathrm{U}$ of heparin, and kept on ice until processed. Leukemic cells were obtained by density separation over a Ficoll-Hypaque gradient and washed 3 times with a solution of HEPES, HBSS, and heparin, as previously described (3).

Determination of MTXPG in bone marrow ALL cells. Intracellular MTXPGs were extracted in a buffered solution (Tris, EDTA, and 2-mercaptoethanol) by boiling $\left(100^{\circ} \mathrm{C}\right.$ for 10 minutes), then frozen at $-80^{\circ} \mathrm{C}$ until analysis. The HPLC separation and the radioenzymatic quantitation of MTX and 6 polyglutamylated metabolites $\left(\mathrm{MTXPG}_{2}\right.$ to $\mathrm{MTXPG}_{7}$ ) were performed as previously described (3). All results were expressed as picomoles of MTX or MTXPG per $10^{9}$ cells.

Pharmacokinetic analysis. The systemic pharmacokinetics of MTX were estimated using a first-order 2-compartment model, using a Bayesian estimation algorithm, as implemented in ADAPT II software (Biomedical Simulations Resource, University of Southern California, Los Angeles, California, USA) (48). The area under the curve from 0 to 48 hours $(\mu \mathrm{M} \times \mathrm{h} / \mathrm{l})$, before the first dose of leucovorin, was calculated by standard trapezoidal methods using the best-fit curve as previously described (3).

Cell cycle distribution. The percentage of ALL cells in S phase was determined in diagnostic bone marrow aspirates in patients for whom an adequate number of cells were available $(n=54)$. Propidium iodide-stained DNA content was measured by flow cytometry using the Coulter EPICS $\mathrm{V}$ flow cytometer (Coulter Electronics Corp.), and the computer program ModFit (Verity Software House Inc.) was used to calculate the percentages of cells in $G_{0} / G_{1}, S$, and $G_{2} / M$ phase, respectively.

RNA extraction and gene expression profiling. Of all 244 patients, 197 patients with sufficient blasts in their bone marrow aspirates to permit RNA isolation from 5 million to 10 million leukemia cells (median $97 \%$ blasts) were available for assessment of gene expression in pretreatment bone marrow ALL cells. Fifty-four of these 197 patients were among the 101 patients for whom MTXPG was measured in ALL cells after HDMTX treatment (Supplemental Figure 2). We extracted high-quality total RNA with TRI Reagent (Molecular Research Center Inc.) from cryopreserved mononuclear cell suspensions from bone marrow at diagnosis. Using methods previously described in detail (47), total RNA was processed and hybridized to the HG-U133A GeneChip oligonucleotide microarray (Affymetrix Inc.; see manufacturer's manual for detailed protocol) (49). Default settings of Microarray Suite software version 5.0 (Affymetrix Inc.) were used to calculate scaled gene expression values.

A total of 53 candidate gene probe sets (representing 32 genes) were selected for the analysis, based on a search of the National Center for Biotechnology Information (50) for "mtx, transport, metabolism, human" and an examination of the Kyoto Encyclopedia of Genes and Genomes (KEGG) pathway database (51) under "Human, one carbon pool by folate," and "Human, folate biosynthesis." Among these candidate genes were also included 3 genes (7 probe sets for 5,10-methylenetetrahydrofolate reductase [MTHFR], phosphoribosyl pyrophosphate amidotransferase [PPAT], and ferritin heavy polypeptide 1 [FTH1] genes) that are known to be of potential importance in folate/antifolate metabolism but were not found in the National Center for Biotechnology Information search or the KEGG database (Supplemental Table 1).

To further establish the validity of gene expression determined by microarray analysis, we performed quantitative real-time RT-PCR in 4 patient samples for 4 genes. The correlation $\left(r^{2}\right)$ between mRNA expression determined by real-time RT-PCR and microarray analyses was $84 \%$ for ATIC, $91 \%$ for TYMS, 75\% for ABCG2, and 88\% for MTHFD1 (Supplemental Figure 3).

Folate pathway gene expression, MTXPG accumulation, and peripheral blast reduction after HDMTX. White blood cell count and manual differential were determined in peripheral blood samples obtained before HDMTX administration and on day 4 after the start of HDMTX therapy. The white blood cell count was measured using a Coulter Counter (model F+STKR; Coulter Electronics Corp.), and the manual differential was performed using Wright-Giemsa stain with Hematek 2000 (Ames Corp.). The peripheral blast count was calculated as the white blood cell count multiplied by the percentage of blasts on a peripheral smear, divided by $100\left(10^{9}\right.$ per liter). The effects of MTX on peripheral blast count were assessed in the 14 patients with BNHD ALL who had a peripheral blood blast count greater than $1 \times 10^{9}$ per liter before starting MTX and from whom gene expression and MTXPG data were available.)

Statistical analysis and bioinformatics. Expression signals were log-transformed. Probe sets called "absent" in over 95\% of 197 patients were not included in the following analysis. Highly correlated probe sets (Pearson correlation coefficient $r>0.4$ ) encoding for the same gene were considered to be measuring 1 transcript and were combined into 1 expression variable by averaging of the standardized signals of each probe set. Probe sets representing the same gene with expression not correlated were considered independent transcripts (i.e., for GART and PPAT; Supplemental Table 1).

Differences in MTX areas under the curve and MTXPG accumulation among the 5 ALL subtypes, as well as differences in gene expression for each transcript among subtypes, were evaluated using the Kruskal-Wallis test and by pairwise comparisons using the Wilcoxon rank sum test. $P$ values from pairwise comparisons were adjusted for multiple testing using Holm's method (52). To further illustrate the differences in gene expression pattern among subtypes, we randomly split the patients into a "training set" (two-thirds of patients) and a "test set" (one-third of patients). We constructed a subtype classifier by SVM based on transcripts associated with ALL subtypes $(P<0.01$, Kruskal-Wallis test) in the training set. We then applied the classifier to patients in the test set to estimate the accuracy for predicting ALL subtype. We performed this procedure 1,000 times to provide the $95 \%$ confidence intervals of estimated accuracy.

We also predicted the MTXPG level for each patient using these candidate transcripts based on leave-one-out cross-validation with SVM. The significance of model-predicted MTXPG compared with the observed MTXPG levels was assessed by multiple linear regression analysis, adjusting for ALL subtypes. Spearman's rank correlation was used to associate MTXPG levels with the expression level of each transcript for patients within ALL subtypes, and the $P$ values were adjusted for multiple testing according to Holm's method (52).

Unsupervised hierarchical clustering with average linkage was used to cluster both patients and transcripts. Pearson correlation and partial correlation adjusting for subtypes were used to evaluate the relationship between 2 individual transcripts. We searched the TRANSFAC 6.0 database, using Match-Public (53), for putative transcription factor binding sites 1,200 bp upstream of those transcripts whose expression was significantly correlated. All statistical analyses were performed in R version 1.8 .1 software (54).

\section{Acknowledgments}

This work was supported in part by NIH grants R37 CA36401 (to W.E. Evans, M.V. Relling, and C.-H. Pui), R01 CA78224 (to W.E. Evans, M.V. Relling, and C.-H. Pui), RO1 CA51001 (to M.V. Relling and C.-H. Pui), U01 GM61393 (to M.V. Relling and W.E. Evans), and RO1 CA71907-07 (to J.R. Downing); Cancer Center Support Grant CA21765; an F.M. Kirby Clinical Research Professorship from the American Cancer Society (to C.-H. Pui); Erwin Schroedinger grants FWF J2110 and J2304 (to L. Kager); and the American Lebanese Syrian Associated Charities. We thank our clinical staff for scrupulous attention to protocol-based patient care; our research nurses, Sheri Ring, Lisa Walters, Terri Kuehner, Margaret Edwards, and Paula Condy; and the patients and their parents for their participation in this study. We also thank Paxton Baker, John Bienvenu, Yaqin Chu, May Chung, Nancy Duran, Natalya Lenchik, Margaret Needham, and Emily Melton for outstanding technical 
assistance; Richard Ashmun for flow cytometric analysis; Deborah French for helpful discussions; and Nancy Kornegay and Mark Wilkinson for computer and database expertise.

Received for publication June 21, 2004, and accepted in revised form October 19, 2004.

1. Pui, C.H., Relling, M.V., and Downing, J.R. 2004. Acute lymphoblastic leukemia. N. Engl. J. Med. 350:1535-1548.

2. Zhao, R., and Goldman, I.D. 2003. Resistance to antifolates. Oncogene. 22:7431-7457.

3. Masson, E., et al. 1996. Accumulation of methotrexate polyglutamates in lymphoblasts is a determinant of antileukemic effects in vivo. A rationale for high-dose methotrexate. J. Clin. Invest. 97:73-80.

4. Curt, G.A., et al. 1985. Determinants of the sensitivity of human small-cell lung cancer cell lines to methotrexate. J. Clin. Invest. 76:1323-1329.

5. Jolivet, J., Cowan, K.H., Curt, G.A., Clendeninn, N.J., and Chabner, B.A. 1983. The pharmacology and clinical use of methotrexate. N. Engl. J. Med. 309:1094-1104.

6. Allegra, C.J., et al. 1985. Enhanced inhibition of thymidylate synthase by methotrexate polyglutamates. J. Biol. Chem. 260:9720-9726.

7. Allegra, C.J., Drake, J.C., Jolivet, J., and Chabner, B.A. 1985. Inhibition of phosphoribosylaminoimidazolecarboxamide transformylase by methotrexate and dihydrofolic acid polyglutamates. Proc Natl. Acad. Sci. U. S. A. 82:4881-4885.

8. Relling, M.V., et al. 1994. Patient characteristics associated with high-risk methotrexate concentrations and toxicity. J. Clin. Oncol. 12:1667-1672.

9. Gorlick, R., et al. 1996. Intrinsic and acquired resistance to methotrexate in acute leukemia. N. Engl.J. Med. 335:1041-1048.

10. Hooijberg, J.H., et al. 1999. Antifolate resistance mediated by the multidrug resistance proteins MRP1 and MRP2. Cancer Res. 59:2532-2535.

11.Zeng, H., Chen, Z.S., Belinsky, M.G., Rea, P.A., and Kruh, G.D. 2001. Transport of methotrexate (MTX) and folates by multidrug resistance protein (MRP) 3 and MRP1: effect of polyglutamylation on MTX transport. Cancer Res. 61:7225-7232.

12. Chen, Z.S., et al. 2002. Analysis of methotrexate and folate transport by multidrug resistance protein 4 (ABCC4): MRP4 is a component of the methotrexate efflux system. Cancer Res. 62:3144-3150.

13. Volk, E.L., and Schneider, E. 2003. Wild-type breast cancer resistance protein (BCRP/ABCG2) is a methotrexate polyglutamate transporter. Cancer Res. 63:5538-5543.

14. Gifford, A.J., et al. 1998. P-glycoprotein-mediated methotrexate resistance in CCRF-CEM sublines deficient in methotrexate accumulation due to a point mutation in the reduced folate carrier gene. Int. J. Cancer. 78:176-181.

15. McGuire, J.J., and Bertino, J.R. 1981. Enzymatic synthesis and function of folylpolyglutamates. $\mathrm{Mol}$. Cell. Biochem. 38(Spec. No.):19-48.

16. Galivan, J., et al. 2000. Glutamyl hydrolase. Pharmacological role and enzymatic characterization. Pharmacol. Ther. 85:207-215.

17. Whitehead, V.M., et al. 1992. Accumulation of high levels of methotrexate polyglutamates in lymphoblasts from children with hyperdiploid (greater than 50 chromosomes) B-lineage acute lymphoblastic leukemia: a Pediatric Oncology Group study. Blood. 80:1316-1323.

18. Whitehead, V.M., et al. 1998. Accumulation of methotrexate polyglutamates, ploidy and trisomies of both chromosomes 4 and 10 in lymphoblasts from children with B-progenitor cell acute lymphoblastic leukemia: a Pediatric Oncology Group Study. Leuk. Lymphoma. 31:507-519.
Address correspondence to: William Evans, St. Jude Children's Research Hospital, 332 North Lauderdale Street, Memphis, Tennessee 38105, USA. Phone: (901) 495-3663; Fax: (901) 525-6869; E-mail: william.evans@stjude.org.

Leo Kager and Meyling Cheok contributed equally to this work.
19. Whitehead, V.M., et al. 2001. The association of the TEL-AML1 chromosomal translocation with the accumulation of methotrexate polyglutamates in lymphoblasts and with ploidy in childhood B-progenitor cell acute lymphoblastic leukemia: a Pediatric Oncology Group study. Lenkemia. 15:1081-1088

20. Synold, T.W., et al. 1994. Blast cell methotrexatepolyglutamate accumulation in vivo differs by lineage, ploidy, and methotrexate dose in acute lymphoblastic leukemia. J. Clin. Invest. 94:1996-2001.

21. Barredo, J.C., et al. 1994. Differences in constitutive and post-methotrexate folylpolyglutamate synthetase activity in B-lineage and T-lineage leukemia. Blood. 84:564-569.

22. Galpin, A.J., et al. 1997. Differences in folylpolyglutamate synthetase and dihydrofolate reductase expression in human B-lineage versus T-lineage leukemic lymphoblasts: mechanisms for lineage differences in methotrexate polyglutamylation and cytotoxicity. Mol. Pharmacol. 52:155-163.

23. Rots, M.G., et al. 1999. Role of folylpolyglutamate synthetase and folylpolyglutamate hydrolase in methotrexate accumulation and polyglutamylation in childhood leukemia. Blood. 93:1677-1683.

24. Zhang, L., et al. 1998. Reduced folate carrier gene expression in childhood acute lymphoblastic leukemia: relationship to immunophenotype and ploidy. Clin. Cancer Res. 4:2169-2177.

25. Belkov, V.M., et al. 1999. Reduced folate carrier expression in acute lymphoblastic leukemia: a mechanism for ploidy but not lineage differences in methotrexate accumulation. Blood. 93:1643-1650.

26. Cheng, Q., et al. 2004. A substrate specific functional polymorphism of human gamma-glutamyl hydrolase alters catalytic activity and methotrexate polyglutamate accumulation in acute lymphoblastic leukaemia cells. Pharmacogenetics. 14:557-567.

27. Suh, J.R., Oppenheim, E.W., Girgis, S., and Stover, P.J. 2000. Purification and properties of a folate-catabolizing enzyme. J. Biol. Chem. 275:35646-35655.

28. Foa, R., et al. 2003. E2A-PBX1 fusion in adult acute lymphoblastic leukaemia: biological and clinical features. Br. J. Haematol. 120:484-487.

29. Crist, W.M., et al. 1990. Poor prognosis of children with pre-B acute lymphoblastic leukemia is associated with the $\mathrm{t}(1 ; 19)(\mathrm{q} 23 ; \mathrm{p} 13)$ : a Pediatric Oncology Group study. Blood. 76:117-122.

30. Raimondi, S.C., et al. 1990. Cytogenetics of pre-Bcell acute lymphoblastic leukemia with emphasis on prognostic implications of the $\mathrm{t}(1 ; 19)$. J. Clin. Oncol. 8:1380-1388.

31. Pui, C.H., et al. 2003. Results of therapy for acute lymphoblastic leukemia in black and white children. JAMA. 290:2001-2007.

32. Trippett, T., et al. 1992. Defective transport as a mechanism of acquired resistance to methotrexate in patients with acute lymphocytic leukemia. Blood. 80:1158-1162.

33. Gorlick, R., et al. 1997. Defective transport is a common mechanism of acquired methotrexate resistance in acute lymphocytic leukemia and is associated with decreased reduced folate carrier expression. Blood. 89:1013-1018.

34. Sierra, E.E., and Goldman, I.D. 1999. Recent advances in the understanding of the mechanism of membrane transport of folates and antifolates. Semin. Oncol. 26:11-23.
35. Schrappe, M., et al. 2000. Improved outcome in childhood acute lymphoblastic leukemia despite reduced use of anthracyclines and cranial radiotherapy: results of trial ALL-BFM 90. GermanAustrian-Swiss ALL-BFM Study Group. Blood. 95:3310-3322.

36. Hooijberg, J.H., et al. 2003. The role of multidrug resistance proteins MRP1, MRP2 and MRP3 in cellular folate homeostasis. Biochem. Pharmacol. 65:765-771.

37. Hooijberg, J.H., et al. 2004. Folate concentration dependent transport activity of the Multidrug Resistance Protein 1 (ABCC1). Biochem. Pharmacol. 67:1541-1548.

38. Assaraf, Y.G., et al. 2003. Loss of multidrug resistance protein 1 expression and folate efflux activity results in a highly concentrative folate transport in human leukemia cells. J. Biol. Chem. 278:6680-6686.

39. Volk, E.L., et al. 2002. Overexpression of wild-type breast cancer resistance protein mediates methotrexate resistance. Cancer Res. 62:5035-5040.

40. Plasschaert, S.L., et al. 2003. The role of breast cancer resistance protein in acute lymphoblastic leukemia. Clin. Cancer Res. 9:5171-5177.

41. DeGregori, J. 2002. The genetics of the E2F family of transcription factors: shared functions and unique roles. Biochim. Biophys. Acta. 1602:131-150.

42. Li, W.W., Fan, J., Hochhauser, D., and Bertino, J.R. 1997. Overexpression of $\mathrm{p} 21$ waf1 leads to increased inhibition of E2F-1 phosphorylation and sensitivity to anticancer drugs in retinoblastoma-negative human sarcoma cells. Cancer Res. 57:2193-2199.

43. Rots, M.G., et al. 2000. mRNA expression levels of methotrexate resistance-related proteins in childhood leukemia as determined by a standardized competitive template-based RT-PCR method. Leukemia. 14:2166-2175.

44. Zaza, G., et al. 2004. Acute lymphoblastic leukemia with TEL-AML1 fusion has lower expression of genes involved in purine metabolism and lower de novo purine synthesis. Blood. 104:1435-1441.

45. Uckun, F.M., et al. 1998. Biology and treatment of childhood T-lineage acute lymphoblastic leukemia. Blood. 91:735-746.

46. Dervieux, T., et al. 2002. De novo purine synthesis inhibition and antileukemic effects of mercaptopurine alone or in combination with methotrexate in vivo. Blood. 100:1240-1247.

47. Ross, M.E., et al. 2003. Classification of pediatric acute lymphoblastic leukemia by gene expression profiling. Blood. 102:2951-2959.

48. D'Argenio, D.Z. 1990. Incorporating prior parameter uncertainty in the design of sampling schedules for pharmacokinetic parameter estimation experiments. Math. Biosci. 99:105-118.

49. Lipshutz, R.J., Fodor, S.P., Gingeras, T.R., and Lockhart, D.J. 1999. High density synthetic oligonucleotide arrays. Nat. Genet. 21:20-24.

50. National Center for Biotechnology Information. http://www.ncbi.nlm.nih.gov.

51. KEGG: Kyoto Encyclopedia of Genes and Genomes. http://www.genome.ad.jp/kegg.

52. Westfall, P.H., Zaykin, D.V., and Young, S.S. 2002. Multiple tests for genetic effects in association studies. Methods Mol. Biol. 184:143-168.

53. Match-Public. http://www.gene-regulation.com/ pub/programs.html\#match.

54. The R project for Statistical Computing. http:// www.r-project.org. 